\title{
A Perspective on Human Exposures to Plastics Additives in Water-Packaging Materials
}

\author{
Syam S. Andra \\ Water and Health Laboratory, Cyprus International Institute for Environmental and Public Health in Association with \\ Harvard School of Public Health, Cyprus University of Technology, Limassol, Cyprus \\ Email: syam.andra@cut.ac.cy
}

Received February 18, 2013; revised March 20, 2013; accepted April 2, 2013

Copyright (C) 2013 Syam S. Andra. This is an open access article distributed under the Creative Commons Attribution License, which permits unrestricted use, distribution, and reproduction in any medium, provided the original work is properly cited.

\begin{abstract}
Plastic and polymer additives (PA) have unique rational advantages for various water and food packaging applications. However, their (bio)chemical natures are recently recognized for their negative human health impacts. The major adverse consequence of these additives in consumer products is in the form of endocrine-disruption related health-downgrades. Such findings still remain underappreciated in most parts globally; part of which could be ascribed to fragmented studies towards better understanding on the occurrence, release and migration, human exposure, epidemiology and risk assessment of PA from packaging materials. In addition there is limited and disconnected dissemination of research findings on PA effects and mitigation measures to society at present. In light of appropriateness of this topic, a trans-disciplinary research agenda is required for addressing exposure routes to PA, human health burden and prevention measures. This perspective article discusses important research questions relating to PA, which try to shed light to a grey scientific area and help increase consumers' awareness and intervention to such exposures.
\end{abstract}

Keywords: Bottled Water; Endocrine Disrupting Chemicals; Food Contact Material; Packaging Material; Plastic Additives; Water Contact Material

\section{Introduction}

Plastic and polymer additives (PA) have unique rational advantages for various water and food packaging applications. Use of PA in every-day consumer products worldwide is estimated to reach $\sim 230$ million tons by 2015 [1]. Polyethylene terephthalate (PET) among all types of plastics is expected to have the highest annual growth rate (11\%) [1]. Bottles for packaging drinkingwater represent one of the most popular uses of plastic and polymer additives. The impact of global stressors, such as, population and consumption, demographic and land-use changes, urbanization, and climate change on water demand and supply dynamics have largely affected the booming growth of the bottled water industry. The bottled water market enjoyed a $25 \%$ global increase in average consumption per capita between 2004 and 2009 [2]. Increases in global population and urbanization along with climate change effects on water supply and availability have been charged with increasing consumer preference towards bottled water in both developed and developing countries. Current estimates showed that United States is the leading consumer of bottled water at about 8500 million gallons contributing to $15.8 \%$ of global consumption, while Mexico tops the global per capita annual bottled water consumption (234 L·capita ${ }^{-1}$ ) [2]. It is noteworthy that 12 out of the top 20 countries leading the global per capita consumption list of bottled water come from the EU [2].

Public concerns related to widespread consumption of bottled water stem from accusations on inadequate sustainability metrics application and conformity to public health standards [3]. It was only recently that the scientific community began to deal with the presence of toxic contaminants and bacteria in the finished water whether initially present in the raw water or as a result of leaching mechanisms. Water contact materials (primarily plastic) have been recently charged with the release of endocrine disrupting compounds (EDCs) into bottled water, such as, bisphenol A (BPA), organo-brominated compounds, perfluorinated compounds, antimony $(\mathrm{Sb})$ and other alkyl phenols like 4-nonylphenol, adipates, phthalates, etc., [3-6]. Despite having useful applications to PA use, their (bio) chemical natures are recently recognized for their negative human health impacts. A major adverse consequence of these additives in consumer products are in the 
form of endocrine-disruption related health-downgrades such as reproductive [7] and thyroid health abnormalities [8], which has exposed poor understanding on their safety and superimposed an urgency to control the potential future risks. The count of occurrence of diseases and disorders from plastics additives is mounting. With the decline in communicable diseases worldwide, chronic diseases from environmental and occupational chemicals is gaining attention. Wittassek et al. [9] review on phthalates provides the magnitude of phthalates usage in industrial and commercial applications and details on magnitude of associated human exposure and health outcomes (in particular male reproductive dysfunctions, also known as phthalate syndrome). Extensive reviews on occurrence and exposures to plastics additives that are widely in use are available for 1) bisphenol A [10,11], 2) phthalates $[9,12,13]$ and 3) polybrominated biphenyl ethers [14-16]. These latest reviews on estimating risks to human health from endocrine-disrupting chemicals (EDC) from plastics additives in packaged containers calls for further research.

While we are identifying new and acknowledging existing research gaps, we propose the need and outlook for more coherent way(s), with input from a range of disciplines, to fill in the knowledge gaps on human exposures to water packaging contact materials. The purpose of this perspective article is not to rehash and/or update the relevant literature; but to present in a nutshell the current research status and knowledge gaps in an attempt to reach a wider inter-disciplinary research community.

\section{Research Status: Current and Next Steps}

The overall hypothesis of community health research on plastics additives (PA) exposure is to understand whether current understanding on plastics additives exposure is sufficient to understand community health risks and to invent prevention measures. This research question puts together in a nutshell the long term quest of environmental and public health researchers for understanding the association between contaminants levels in exposure environmental media, biomonitored levels in human body tissues/fluids, and disease endpoints. The research questions sketched here, proactively, is expected to promote sharing of research ideas and help combine and recombine in novel ways to evolve into new research areas; which gets quickly filtered in research collaborations.

\subsection{Is Release of Plastics Additives to Contained Water Ongoing over Time or Are There Special Circumstances That Enhance These Processes?}

\subsubsection{Current Status}

Migration is a term used for release of both intention- ally and non-intentionally added substances to the polymers; while induced leaching (also referred to as "release") occurs from degradation of polymers under physical and environmental conditions such as exposure to UV light, heat, polymer age etc. [17]. Consumer concerns take the form of episodic chemical leaching from water-contact materials (WCM) [18], but these are often considered unfounded [19]. Examples of chemical leaching from bottled water are: antimony [20-22], bisphenol A [23], phthalates [24], adipates [25], and 4-nonylphenol [6]. Leaching of PA from container material to contained food and water products by diffusion [26] and other physico-chemical processes are reported for bisphenol A [27-31] and phthalates [28,32]. Migration of PA, such as BPA and 4-nonylphenol, was reported occurring from container material to bottled water [33]. Based on BPA migration, the calculated total daily intake estimate was $0.00004 \mathrm{mg} \cdot \mathrm{kg}^{-1}$ body weight $\cdot \mathrm{day}^{-1}$ [33], which was significantly lower than the total daily intake limit of 0.05 $\mathrm{mg} \cdot \mathrm{kg}^{-1}$ body weight $\cdot \mathrm{day}^{-1}$ set by CEF/AMU [34]. However, despite the observed total daily intake for BPA in polycarbonate bottled water being lower than the set total daily intake limit, is it prudent to consider it safe? This could be supported by the observation that BPA was present at higher levels in human matrices, which are above those that could induce harmful effects in both in vitro and in vivo tests [10]. In the EU, a migration limit of $0.3 \mathrm{mg}$ di-n-butyl phthalate per $\mathrm{kg}$ food stimulant and $1.5 \mathrm{mg}$ di-2-ethylhexyl phthalate per $\mathrm{kg}$ food stimulant applies $[9,35]$. Triclosan was one intentionally added food contact material as a way to extend food shelf life, the use of which was unauthorized in US and withdrawn from use in the EU [17]. Environmental factors such as exposure to sunlight for ten weeks increased BPA levels in bottled water [28]. In addition, PA leaching into food and water under actual conditions were much higher in comparison to standardized migration tests using distilled water [36]. However there are several components of these polymers and several other factors that promote their release that are yet to be tested in migration studies.

A common practice of bottle reuse for refilling tap water has shown an increase of antimony release with each reuse cycle [22]. Frequency of reuse has never been tested before with respect to its influence on chemical leaching from drinking-water plastic containers. Our study showed that frequency of reuse exerted greater influence on $\mathrm{Sb}$ and $\mathrm{Br}$ leaching from PET and PC containers when compared with that attributed to UV exposure duration and temperature. Our cost-effective experimental approach in studying main and interaction effects of three major tested factors influencing chemical leaching enables us to classify the studied factors according to their contribution to chemical leaching from frequency of reuse $>$ UV exposure duration $>$ tempera- 
ture [22]. This study illustrated the importance of so far unaccounted factor, i.e., frequency of bottle reuse in various everyday activities that could enhance chemical leaching. Antimony leaching from PET seems to be the most concerning observation, while the reasons and health implications behind leaching of soluble $\mathrm{Br}$ (associated with organo bromine compounds, such as PBDE) in this study are yet to be determined.

\subsubsection{Next Steps}

Assessment of migrants levels using partition models is required taking into consideration the complexity of packed food and water matrices compared to the matrices in current use for testing. It is important to understand the factors that affect migration of PA in mixtures at low concentrations rather than single chemicals. So far food contact materials toxicity assessment is performed using a single chemical approach, while it is shown that chemicals migrate in groups as a mixture having whole effects that can't be explained by toxicological aspects of one or few known PA [37,38].

\subsection{Do Concentrations of Plastics Additives in Body Tissues/Fluids Indicate the Stage and Extent of Disease/Disorder Condition?}

\subsubsection{Current Status}

Body burden of PA is widely reviewed recently by Koch and Calafat [12]. Non-communicable diseases, such as cancer, diabetes, cardiovascular and chronic respiratory diseases are predicted to cost more than US\$ 30 trillion, representing $48 \%$ of global gross domestic product in 2010 over the next 20 years [39]. Such comprehensive disease predictive models often assume a constant number of annual new incidence cases for a specific outcome, but often this is not the case. An illustrating example is thyroid cancer, which appears as the outcome with the highest change in annual excess number of new cases in the developed world. Endocrine-disrupting chemicals, or particularly, thyroid-disrupting chemicals are synthetic chemicals used in everyday consumer products mimicking or antagonizing natural thyroid hormone processes [8]. While polybrominated diphenyl ethers (PBDE) levels have been quantified in several subgroups and biomarkers for thyroid health were measured, no significant trend in dose relationships with thyroid hormones levels were identified [8]. Similarly, almost all studies that were mentioned in the exclusive reviews on 1) bisphenol A $[10,11], 2)$ phthalates $[9,12,13]$ and 3) polybrominated biphenyl ethers [14-16], attempted quantifying body burden of each PA of their study interest; but did not correlate them with a disease endpoint.

\subsubsection{Next Steps}

Quantification of disease incidence or a physiological response in terms of extent of exposure is very fundamental in making precise risk assessments, which at present is an inadequate science that can and should be addressed. Focus should be on higher prevalence specific disorders such as thyroid disruption, fertility rate decline etc; as well as on general health status such as endocrine disruption that impacts number of physiological mechanisms downstream. How relevant is a smaller or larger body burden of a given PA related to a disease endpoint? Answers are still needed on what the body burden numbers mean in a human toxicological perspective. Additional studies are required to pinpoint disease endpoints for each or a mixture of PA exposures. There is also a need for longitudinal studies in understanding changing reproductive functions in males, increasing in thyroid disruption in females, and pronounced endocrine disruption outcomes in young adults.

\subsection{Do a Certain Set of Populations Tend to Be More Exposed to Plastics Additives and/or Are There Sensitive Life Stages to Such Exposures?}

\subsubsection{Current Status}

Children are one hundred percent of our community future, and it seems less than one percent understanding is attained on their direct and/or indirect exposures to plastics additives and subsequent health outcomes. It is becoming clearer that variations in responses to PA exposure among populations is enormous, which differs based on genetic makeup, ethnical and cultural factors, socioeconomic-nutrition-occupational variables, age and gender differences, etc. Attention needs to be paid to the certain observation that some individuals, within the general population, show excess body levels for one or more phthalates above the recommended tolerable daily intake levels [9]. Phthalates exposure is high in children compared with adults [9]. Children had maximum exposure to phthalates compared to adults, and are speculated to experience phthalates exposure from playing with toys. A maximum daily intake of about $400 \mu \mathrm{g} \cdot \mathrm{kg}^{-1} \cdot \mathrm{day}^{-1}$ for di-2-ethylhexyl phthalate was found in two to four children in Germany [9]; while the total daily intake limit value for di-2-ethylhexyl phthalate is $50 \mu \mathrm{g} \cdot \mathrm{kg}^{-1} \cdot \mathrm{day}^{-1}$ in Europe [40,41]. Premature neonates in intensive care units are exposed to very high doses of phthalates (in particular, di-2-ethylhexyl phthalate) [42], and exposure to diethyl phthalate and di-n-butyl phthalate containing tables from long term medication in adults [43].

Metabolically dysfunctional physiological states such as obesity, insulin resistance, diabetes etc are found correlated with environmental toxicants exposures and as well as from toxicant susceptibility factors. In case of PBDEs, child-bearing age women and pregnant mothers are highly susceptible compared with adult men with re- 
spect to thyroid health as indicated by respective thyroid hormones levels [8]. Research provides evidence that the metabolic programming that occurs in pre-and post-natal period can be modified by environmental exposures. A small volunteer group of people studying or working in the Cyprus University of Technology were recruited to study their water use and sources habits via administering them a questionnaire while also a spot urine sample was collected. It was shown that there is 1) a major contribution of unexplored routes of bottled water use in the form of cold and hot beverages that increased the cumulative per capita water consumption significantly, which translates to a thus far under-estimation of health risk assessment $[44,45] ; 2)$ a significant $(p=0.02)$ positive association between daily water consumption from polyethylene terephthalate (PET) bottles and urinary $\mathrm{Sb}$, a biomarker for exposures to plastics additives in PET bottled water [44]; and 3) a similar significant $(p=0.02)$ positive association between daily water consumption from polycarbonate (PC) bottles and urinary bisphenol A (BPA), a biomarker for exposures to plastics additives in PC bottled water [45].

Reports are available on transgenerational persistence of a disease from chemical exposures on an individual's epigenome [46]. Exposures to PA in the early life stages such as pre-natal through young ages were shown leading to later life or next generational health effects as a carry-over effect. Examples of prenatal exposures to such PA having postnatal effects, such as pregnant mothers' exposure to: 1) phthalates impacting child's mental and behavioral development [47], 2) BPA resulting in decreased thyroid-stimulating hormone and affecting thyroid health in male infants [48], and 3) brominated flame retardants adversely affecting child neurodevelopment [49]. Co-exposures to different endocrine disrupting and other chemical contaminants in drinking water should be also prioritized by focusing on mixtures of water contaminants and their contribution to biologically relevant exposures. Community studies thus far points out which PA have emerged as key health imposters. Clear and present dangers of endocrine disruption are breast cancer and endometriosis in females, testicular and prostate cancer in males, abnormal sexual development and lowered fertility in both genders; while known trans-generational effects were immune suppression and neurobehavioral effects in infants and children.

\subsubsection{Next Steps}

There is a need for identifying early-stage as well as critical-stage exposure biomarkers that goes beyond associations derived from literature, and helps in testing at individual level and live up to their promise and potential. Attention should be focused on individual practices, life stage, socio-economic-occupational status, and geographi- cal location of maximum exposure in identifying variables for high level PA exposures and/or greater fraction of exposed subgroups. Identifying critical time windows of sensitivity and susceptibility of subgroups to each plastic additive and as well in a mixture helps in designing intervention measures. Do early stage exposures change the future genome and do exposures in a previous generation shapes the future genomes? Understanding the PA exposures during development (pre- and postnatal) will help understand the genetic contribution from shared gene loci among different endocrine disruption outcomes. There is also a need to understand the complex interactions between PA exposure media, individuals' vulnerability factors, disease development, and time to disease appearance for pin pointing the susceptible groups. This helps to avoid discriminatory actions such as "blame the victim" trends based on understanding the underlying causes rather single out the susceptible groups based on socio-economic-cultural factors.

Dietary interventions can buffer PA exposure and associated health risks, which raises the question of "how can safe and green packaging materials fit into the exposure mitigation paradigm?" Short term exposures are evident in infants and children, while a longer term ones were not seen until adulthood. Not only pain and human suffering associated with PA exposure in short and long term effects, but also economic impact of infants and children health is a substantial burden to the community. Since the prenatal time window seems most vulnerable developmental phase, it seems studying prenatal exposures will help in understanding exposome of PA chemical mixtures and as well where in intervention measures can prevent or minimize exposures, resulting in a greatest cost to benefit ratio for the society.

\subsection{Is a Continuous and Cumulative Effect from a Single Plastics Additive Exposure More Harmful Compared to a Snap-Shot Mixtures Exposure?}

\subsubsection{Current Status}

Chemicals having a common disease endpoint, act synergistically when exposed in mixtures. Studies show that cumulative exposures to phthalates tend to be more harmful compared to a single exposure in animal studies, and more potent in a synergistic way when exposed along other endocrine disruptors compared to single class of chemicals exposure. At least fifty chemicals that were used as food contact materials exhibited endocrine disruption properties [5], and studies are lacking on understanding whether the disease outcome is more influenced by a brief exposure from a mixture of these or by a long term cumulative exposure from a single additive. Despite availability of decent number of studies on exposure routes to the noted PA and on their levels in human ma- 
trices, not much information on factors which guide the magnitude of such exposures in mixtures [16]. However studies are still lacking for the new and emerging plastics additives. Animal studies are becoming available for toxicological effects of other brominated flame retardants (other BFRs), but there are only limited human studies [16].

While much scientific attention has been drawn upon single contaminant leaching from plastic bottled water, no study has paid attention to concomitant leaching of two or more contaminants within the same bottle. In a recent study, both $\mathrm{Sb}$ and brominated compounds, as expressed by total soluble $\mathrm{Br}$ measurements, including those for polybrominated biphenyl ethers (PBDE) were measured in bottled water [50] in a representative basket survey sampling of bottled water in randomly selected Boston, MA, USA supermarkets. Different bottled water classes were sampled ranging from: 1) non-carbonated (NCR), 2) carbonated (CR), and 3) non-carbonated and enriched (NCRE). In addition, different bottled water plastic materials were sampled ranging from polyethylene terephthalate (PET), high-density polyethylene (HDPE), and polycarbonate (PC), and polystyrene (PS). The objectives of this study were: 1) determine the effects of plastic material (PET, HDPE, PC, and PS), bottled water classes (non-carbonated, carbonated, and noncarbonated and enriched), and storage time on the simultaneous co-leaching patterns of antimony and brominated compounds from bottled water, and 2) qualitatively characterize the type of brominated compounds leaching from plastic bottled water. Results showed that average $\mathrm{Br}$ and $\mathrm{Sb}$ concentrations after 60-days of storage followed the order of $\mathrm{NCR}<\mathrm{CR}=\mathrm{NCRE}$, and $\mathrm{NCR}<\mathrm{CR}$ $<$ NCRE, respectively, suggesting that the presence of dissolved carbon dioxide in CR samples coupled to additions of flavors and color to NCRE could explain the elevated leaching of $\mathrm{Br}$ and $\mathrm{Sb}$ [50]. Among samples with the highest soluble $\mathrm{Br}$ concentrations, BDE-209 congener was qualitatively confirmed in three out of four bottled water samples. The PC, HDPE, and PS samples exhibited significantly $(\mathrm{p}<0.05)$ lower $\mathrm{Sb}$ and $\mathrm{Br}$ leaching than PET [50]. A group of PA can be occurring in bottled water at any given time, and the composition varies with the chemical nature of the packaging material.

\subsubsection{Next Steps}

Little or no information exists on exposure and dose response for chemical mixtures. Meta-analyses of literature data existing for identification and confirmation of trends in exposure risk factors, target life stages and subgroups, and interventions (if any) are necessary. Only few of the several PA and associated health outcomes have good epidemiological studies, which thus prohibit obtaining a grasp on the complete magnitude of the problem, making creation of effective intervention measures difficult. Epicohorts are needed to rapidly test and identify potential biomarkers for single and multiple stressors. There is also a need for in vitro and/or in vivo studies to study responses from single vs. multiple PA exposure, to look at the responsome based on phenotypic or chemical markers, to compare differences in metabolome, etc. Human studies are more needed, while animal studies can be used in conjunction and complementation.

\subsection{Does an Understanding on Exposure Processes Require a Single Science Focus or Multi-Disciplinary Efforts?}

\subsubsection{Current Status}

It is widely acknowledged now that although many PA are linked to endocrine disruption and spiral-down symptoms, each contribution may not be of equal contribution to end outcome. Cumulative disease burden occurs when PA is exposed and acts together in mixtures and even then exposure science alone may not explain the entire process. Exposure science is becoming a weak link in environmental health sciences arena and thus hindering progress in gene-environment science. Systems biology helps in taking an integrative approach using animal systems, individuals, subpopulations, and communities in delineating PA exposures and effects. Exposome on the other hand captures and integrates the complex multiple exposures across domains and presents a spatial and temporal view on subjects' susceptibility. Exposome also helps in overcoming the traditional "looking under the lamppost" approaches. Connecting plastics contact materials exposure to disease through the study of exposome and metabolome approaches is an opportunity and important overarching goal to attain today.

\subsubsection{Next Steps}

Researchers must seek collaborations to induce integration of technologies. Identifying apt early stage biomarkers with coordinated trans-disciplinary efforts helps in taking public health science from "cure" approach to the next level of predicting, preventing, and personalized treatment. There is a need to understand why there is common exposure intensity but different susceptibility of subgroups. So far there has been a lack of integration of cross-disciplinary platforms, lack of exposure quantification across age groups and subpopulations, lack of comparisons across mixtures exposure, and lack of informatics tools to pin point out specific and as well generic biomarkers for PA exposure. Bioinformatics tools are necessary for cross platform identification, validation, quantification, optimization, and integration of PA exposure biomarkers. Furthermore, there is a need for bio- 
markers to work back in understanding exposome but also responsome (responsome denotes changes in observable/measurable biological phenomena from exposures). Metabolome approaches helps in finding key areas to apply translation strategies. With an exposome approach, a cohort study will help in monitoring the entire processes between exposure occurrence and disease appearance, and also helps in providing a conceptual framework on exposure-response relationships and toxicology.

Cohort studies should measure methods to assess exposure which happened years ago, relate current exposures with future outcomes, and also promote development of low-cost intervention measures that can be assessed repeatedly and as well in large numbers. Integrated exposure biology should become "open sourced" with information and databases shared that is not or yet to be published. Integrated exposure biology is intended to provide measures of toxic materials exposures, dietary intake, socio-economic and psychosocial status of an individual and be able to link those measures of an individual environment to changes in body physiological pathways. Exposure biology should provide a snapshot of the exposome and as well helps in teasing out the interaction between exposure variables and human responses. An important step would be to continue validation of new prototypes and establish commercial collaborations for making them broadly available. Systems biology facilitates data handling, analysis, and interpretation of how human pathway-based response relates to exposures doses.

\section{Conclusions}

Consumers enjoy numerous choices of plastic materials to temporarily store water or food items before consumption but this convenience is often not accompanied by appropriate precautionary explanation of possible health threats. This may be particularly true in the case of PA found in numerous water-contact materials that could leach under certain environmental conditions, but science associated with their possible human health effects is ill defined. The recent reports of common environmental processes (i.e., frequency of bottle reuse, temperature, UV exposure) maximizing the leaching of plasticizers and other chemicals into packaged water highlight a neglected and much underreported exposure source that needs to be included in future exposure assessment studies and derivations of acceptable daily PA intake estimates.

Initiatives towards creating and updating epidemiological survey databases for global researches access to information on case and control subjects' details, analytical and statistical tools used, and health outcomes and assessments made will help in developing measures at several stages such as exposure reduction interventions, dietary interventions, medical interventions, and policy interventions. Large scale epidemiological/GIS based studies are required to identify most impacted risk groups and as well large scale multi-group/multi-location research groups are required to provide high level evidence and insight into the exposure epidemiology and interventions programs. There is a need for 1) global bio-banking and miniaturization of epidemiological study samples for further use is emphasized, 2) an international task force on prenatal exposures and disease outcomes, 3) interdisciplinary research groups for better understanding multi-variable nature of prenatal exposures, infant and children disorders, and adult age diseases, 4) trans-disciplinary groups should consist of environmental scientists, toxicologists, epidemiologists, clinicians, endocrinologists, nutritionists and community educators, 5) creating enhanced screening tools for environmental exposures in pregnant women, and 6) more systematic worldwide cohort needs to be established that follows a defined set of optimized and authentic protocols in samples collection, analysis and data interpretation; rather monitoring a small set of chemicals in a small set of subjects in a small set if geography using inconsistent protocols.

Green chemists should create new and benign chemicals, based on access to toxicological information, that have zero endocrine disruption function. When introducing a new chemical for usage in water and food contact materials, they need to be tested not only for mutagenicity and genotoxicity, but also for endocrine disruption by using appropriate in vitro and/or in vivo testing. However chemists need to be trained in toxicological and environmental health sciences for cross-discipline communication for understanding PA toxicological issues and databases. Tests need to be actionable, economical, sustainable, reproducible, upgradeable and transparent. There is a need for an increase in public awareness and hence pressure to change policy decisions as well as to find ways for better dissemination of environmental health education to the communities. Communication research in collaboration with behavioral and social science researchers, sociologists, journalists, non-profit government organizations can improve providing insights on PA exposure and avoidance measures. Open access online databases should be available that displays all the chemicals being used as PA in food and water contact materials for the general public interest. There is a need to create an easy-access electronic database for environmental health assessment and health education literature particularly for pregnant women. Young adults should be reached via twitter or other social network sites. Either or each of the approaches should lead to validated intervention measures to prevent (in the first place) or 
mitigate (if necessary) the exposures and risks from PA.

It is inevitable that such approaches may only be successful if, and only if, collaboration opportunities are established for scientific fields and expertise, such as, environmental chemistry, engineering, exposure science, epidemiologists, risk assessors, social and humanities science researchers and policy makers.

\section{Acknowledgements}

I thank my advisor Dr. Konstantinos C. Makris (Assistant Professor of Environmental Health), Cyprus International Institute for Environmental and Public Health in association with Harvard School of Public Health, Cyprus University of Technology, in making this work possible. I also thank Dr. Helen Bridle, Heriot-Watt University, for editing the special issue papers. Finally, I thank the European Science Foundation (ESF) for giving me an opportunity as an Early Career Researcher to present our work at the ESF Junior Summit "Water: Unite and Divide, Interdisciplinary approaches for a sustainable future", Stresa, Lago Maggiore, Italy (27-30 August 2012).

\section{REFERENCES}

[1] CIPET (Central Institute of Plastics and Engineering Technology), "Plastics Industry_Statistics," 2010. http://cipet.gov.in/plastics_statistics.html

[2] J. G. Rodwan Jr., "Bottled Water 2009," International Bottled Water Association," 2010. http://www.bottledwater.org/files/2009BWstats.pdf

[3] C. Z. Yang, S. I. Yaniger, V. C. Jordan, D. J. Klein and G. D. Bittner, "Most Plastic Products Release Estrogenic Chemicals: A Potential Health Problem that Can Be Solved," Environmental Health Perspectives, Vol. 119, No. 7, 2011, pp. 989-996. doi:10.1289/ehp.1003220

[4] J. L. Carwile, H. T. Luu, L. S. Bassett, D. A. Driscoll, C. Yuan, J. Y. Chang, X. Ye, A. M. Calafat and K. B. Michels, "Polycarbonate Bottle Use and Urinary Bisphenol A Concentrations," Environmental Health Perspectives, Vol. 117, No. 9, 2009, pp. 1368-1372.

[5] J. Muncke, "Exposure to Endocrine Disrupting Compounds via the Food Chain: Is Packaging a Relevant Source?" Science of Total Environment, Vol. 407, No. 16, 2009, pp. 4549-4559. doi:10.1016/j.scitotenv.2009.05.006

[6] D. Amiridou and D. Voutsa, "Alkylphenols and Phthalates in Bottled Waters," Journal of Hazardous Materials, Vol. 185, No. 1, 2011, pp. 281-288. doi:10.1016/j.jhazmat.2010.09.031

[7] T. T. Schug, A. Janesick, B. Blumberg and J. J. Heindel, "Endocrine Disrupting Chemicals and Disease Susceptibility," Journal of Steroid Biochemistry and Molecular Biology, Vol. 127, No. 3-5, 2011, pp. 204-215. doi:10.1016/j.jsbmb.2011.08.007

[8] S. S. Andra and K. C. Makris, "Thyroid Disrupting Chemicals in Plastic Additives and Thyroid Health," Journal of Environmental Science and Health. Part C, Environ- mental Carcinogenesis \& Ecotoxicology, Vol. 30, No. 2, 2012, pp. 107-151.

[9] M. Wittassek, H. M. Koch, J. Angerer and T. Brüning, "Assessing Exposure to Phthalates-The Human Biomonitoring Approach," Molecular Nutrition \& Food Research, Vol. 55, No. 1, 2011, pp. 7-31.

doi:10.1002/mnfr.201000121

[10] L. N. Vandenberg, R. Hauser, M. Marcus, N. Olea and W. V. Welshons, "Human Exposure to Bisphenol A (BPA)," Reproductive Toxicology, Vol. 24, No. 2, 2007, pp. 139177. doi:10.1016/j.reprotox.2007.07.010

[11] A. G. Asimakopoulos, N. S. Thomaidis and M. A. Koupparis, "Recent Trends in Biomonitoring of Bisphenol A, 4-T-Octylphenol, and 4-Nonylphenol," Toxicology Letters, Vol. 210, No. 2, 2012, pp. 141-154. doi:10.1016/j.toxlet.2011.07.032

[12] H. M. Koch and A. M. Calafat, "Human Body Burdens of Chemicals Used in Plastic Manufacture," Philosophical Transactions of the Royal Society B: Biological Sciences, Vol. 364, No. 1526, 2009, pp. 2063-2078. doi:10.1098/rstb.2008.0208

[13] G. Saravanabhavan and J. Murray, "Human Biological Monitoring of Diisononyl Phthalate and Diisodecyl Phthalate: A Review," Journal of Environmental and Public Health, Vol. 2012, 2012, p. 11. doi:10.1155/2012/810501

[14] L. G. Costa, G. Giordano, S. Tagliaferri, A. Caglieri and A. Mutti, "Polybrominated Diphenyl Ether (PBDE) Flame Retardants: Environmental Contamination, Human Body Burden and Potential Adverse Health Effects," Acta BioMedica, Vol. 79, No. 3, 2008, pp. 172-183.

[15] M. Frederiksen, K. Vorkamp, M. Thomsen and L. E. Knudsen, "Human Internal and External Exposure to PBDEs-A Review of Levels and Sources," International Journal of Hygiene and Environmental Health, Vol. 212, No. 2, 2009, pp. 109-134. doi:10.1016/j.ijheh.2008.04.005

[16] A. Covaci, S. Harrad, M. A. Abdallah, N. Ali, R. J. Law, D. Herzke and C. A. de Wit, "Novel Brominated Flame Retardants: A Review of Their Analysis, Environmental Fate and Behaviour," Environment International, Vol. 37, No. 2, 2011, pp. 532-556. doi:10.1016/j.envint.2010.11.007

[17] J. Muncke, "Endocrine Disrupting Chemicals and Other Substances of Concern in Food Contact Materials: An Updated Review of Exposure, Effect and Risk Assessment," Journal of Steroid Biochemistry and Molecular Biology, Vol. 127, No. 1-2, 2011, pp. 118-127. doi:10.1016/j.jsbmb.2010.10.004

[18] L. Sax, "Polyethylene Terephthalate May Yield Endocrine Disruptors," Environmental Health Perspectives, Vol. 118, No. 4, 2010, pp. 445-448. doi:10.1289/ehp.0901253

[19] IBWA (International Bottled Water Association), "Bottled Water Facts: IBWA Corrects EWG's Faulty Report," Bottled Water Reporter, 2011.

http://www.nxtbook.com/ygsreprints/IBWA/g17910ibwa/ \#/8

[20] W. Shotyk and M. Krachler, "Contamination of Bottled Waters with Antimony Leaching from Polyethylene Tere- 
phthalate (PET) Increases upon Storage," Environmental Science and Technology, Vol. 41, No. 5, 2007, pp. 1560-1563. doi:10.1021/es061511+

[21] P. Westerhoff, P. Prapaipong, E. Shock and A. Hillaireau, "Antimony Leaching from Polyethylene Terephthalate (PET) Plastic Used for Bottled Drinking Water," Water Research, Vol. 42, No. 3, 2008, pp. 551-556. doi:10.1016/j.watres.2007.07.048

[22] S. S. Andra, K. C. Makris and J. P. Shine, "Frequency of Use Controls Chemical Leaching from Drinking-Water Containers Subject to Disinfection," Water Research, Vol. 45, No. 20, 2011, pp. 6677-6687. doi:10.1016/j.watres.2011.10.001

[23] X. L. Cao and J. Corriveau, "Migration of Bisphenol A from Polycarbonate Baby and Water Bottles into Water under Severe Conditions," Journal of Agricultural and Food Chemistry, Vol. 56, No. 15, 2008, pp. 6378-6381. doi:10.1021/jf800870b

[24] S. V. Leivadara, A. D. Nikolaou and T. D. Lekkas, "Determination of Organic Compounds in Bottled Waters," Food Chemistry, Vol. 108, No. 1, 2008, pp. 277-286. doi:10.1016/j.foodchem.2007.10.031

[25] P. Schmid, M. Kohle, R. Meierhofer, S. Luzi and M. Wegelin, "Does the Reuse of PET Bottles during Solar Water Disinfection Pose a Health Risk Due to the Migration of Plasticisers and Other Chemicals into the Water?" Water Research, Vol. 42, No. 20, 2008, pp. 5054-5060. doi:10.1016/i.watres.2008.09.025

[26] D. Biscardi, S. Monarca, R. De Fusco R, F. Senatore, P. Poli, A. Buschini, C. Rossi and C. Zani, "Evaluation of the Migration of Mutagens/Carcinogens from PET Bottles into Mineral Water by Tradescantia/Micronuclei Test, Comet Assay on Leukocytes and GC/MS," Science of Total Environment, Vol. 302, No. 1-3, 2003, pp. 101-108. doi:10.1016/S0048-9697(02)00349-2

[27] J. E. Biles, T. P. McNeal, T. H. Begley and H. C. Hollifield, "Determination of Bisphenol-A in Reusable Polycarbonate Food-Contact Plastics and Migration to FoodSimulating Liquids," Journal of Agricultural and Food Chemistry, Vol. 45, No. 9, 1997, pp. 3541-3544. doi: $10.1021 / \mathrm{jf} 970072 \mathrm{i}$

[28] N. Casajuana and S. Lacorte, "Presence and Release of Phthalic Esters and Other Endocrine Disrupting Compounds in Drinking Water," Chromatographia, Vol. 57, No. 910, 2003, pp. 649-655. doi:10.1007/BF02491744

[29] J. E. Loyo-Rosales, G. C. Rosales-Rivera, A. M. Lynch, C. P. Rice and A. Torrents, "Migration of Nonylphenol from Plastic Containers to Water and a Milk Surrogate," Journal of Agricultural and Food Chemistry, Vol. 52, No. 7, 2004, pp. 2016-2020. doi:10.1021/jf0345696

[30] H. H. Le, E. M. Carlson, J. P. Chua and S. M. Belcher, "Bisphenol A Is Released from Polycarbonate Drinking Bottles and Mimics the Neurotoxic Actions of Estrogen in Developing Cerebellar Neurons," Toxicology Letters, Vol. 176, No. 2, 2008, pp. 149-156. doi:10.1016/i.toxlet.2007.11.001

[31] H. Gallart-Ayala, E. Moyano and M. T. Galceran, "Analysis of Bisphenols in Soft Drinks by On-Line Solid Phase Extraction Fast Liquid Chromatography-Tandem Mass
Spectrometry," Analytica Chimica Acta, Vol. 683, No. 2, 2011, pp. 227-233. doi:10.1016/j.aca.2010.10.034

[32] A. Penalver, E. Pocurull, F. Borrull and R. M. Marce, "Determination of Phthalate Esters in Water Samples by Solid-Phase Microextraction and Gas Chromatography with Mass Spectrometric Detection," Journal of Chromatography A, Vol. 872, No. 1-2, 2000, pp. 191-201. doi:10.1016/S0021-9673(99)01284-4

[33] A. Guart, F. Bono-Blay, A. Borrell and S. Lacorte, "Migration of Plasticizers, Phthalates, Bisphenol A and Alkylphenols from Plastic Containers and Evaluation of Risk," Food Additives \& Contaminants. Part A, Chemistry, Analysis, Control, Exposure and Risk Assessment, Vol. 28, No. 5, 2011, pp. 676-685. doi: $10.1080 / 19440049.2011 .555845$

[34] CEF/AMU, "Statement of EFSA on a Study Associating Bisphenol A with Medical Disorders," The EFSA Journal, Vol. 838, No. 22, 2008, pp. 1-3.

[35] EU, Commission Directive 2007/19/EC of 30 March 2007 Amending Directive 2002/72/EC Relating to Plastic Materials and Articles Intended to Come into Contact with Food and Council Directive 85/572/EEC Laying Down the List of Simulants to Be Used for Testing Migration of Constituents of Plastic Materials and Articles Intended to Come into Contact with Foodstuffs, 2007.

[36] N. von Goetz, M. Wormuth, M. Scheringer and K. Hungerbühler, "Bisphenol A: How the Most Relevant Exposure Sources Contribute to Total Consumer Exposure," Risk Analysis, Vol. 30, No. 3, 2010, pp. 473-487. doi:10.1111/j.1539-6924.2009.01345.x

[37] M. Wagner and J. Oehlmann, "Endocrine Disruptors in Bottled Mineral Water: Total Estrogenic Burden and Migration from Plastic Bottles," Environmental Science and Pollution Research, Vol. 16, No. 3, 2009, pp. 278-286. doi:10.1007/s11356-009-0107-7

[38] U. Honkalampi-Hamalainen, E. L. Bradley, L. Castle, I. Severin, L. Dahbi, O. Dahlman, J.-C. Lhuguenot, M. A. Andersson, P. Hakulinen, D. Hoornstra, J. Maki-Paakkanen, M. Salkinoja-Salonen, L. Turco, A. Stammati, F. Zucco, A. Weber and A. von Wright, "Safety Evaluation of Food Contact Paper and Board Using Chemical Tests and in Vitro Bioassays: Role of Known and Unknown Substances," Food Additives \& Contaminants. Part A, Chemistry, Analysis, Control, Exposure and Risk Assessment, Vol. 27, No. 3, 2010, pp. 1-10.

[39] D. E. Bloom, E. T. Cafiero, E. Jané-Lopis, S. AbrahamsGessel, L. R. Bloom, S. Fathima, et al. "The Global Economic Burden of Non-communicable Diseases," 2011. http://www3.weforum.org/docs/WEF_Harvard_HE_Glob alEconomicBurdenNonCommunicableDiseases_2011.pdf

[40] M. Wittassek, G. A. Wiesmüller, H. M. Koch, R. Eckard, L. Dobler, J. Müller, J. Angerer and C. Schlüter, "Internal Phthalate Exposure over the Last Two Decades-A Retrospective Human Biomonitoring Study," International Journal of Hygiene and Environmental Health, Vol. 210, No. 3-4, 2007, pp. 319-333. doi:10.1016/j.ijheh.2007.01.037

[41] ESFA (European Food Safety Authority), "Opinion of the Scientific Panel on Food Additives, Flavourings, Proc- 
essing Aids and Materials in Contact with Food (AFC) on a Request from the Commission Related to Bis(2-ethylhexyl)phthalate (DEHP) for Use in Food Contact Materials," 2005 .

http://www.efsa.europa.eu/en/scdocs/scdoc/243.htm

[42] J. Weuve, B. N. Sanchez, A. M. Calafat, T. Schettler, R. A. Green, H. Hu, et al., "Exposure to Phthalates in Neonatal Intensive Care Unit Infants: Urinary Concentrations of Monoesters and Oxidative Metabolites," Environmental Health Perspectives, Vol. 114, No. 9, 2006, pp. 1424-1431. doi:10.1289/ehp.8926

[43] R. Hauser, S. Duty, L. Godfrey-Bailey and A. M. Calafat, "Medications as a Source of Human Exposure to Phthalates," Environmental Health Perspectives, Vol. 112, No. 6, 2004, pp. 751-753. doi:10.1289/ehp.6804

[44] K. C. Makris, S. S. Andra, L. Herrick, C. A. Christophi, S. A. Snyder and R. Hauser, "Association of Drinking-Water Source and Use Characteristics with Urinary Antimony Concentrations," Journal of Exposure Science and Environmental Epidemiology, Vol. 23, No. 2, 2013, pp. 120127. doi:10.1038/jes.2012.104

[45] K. C. Makris, S. S. Andra, A. Jia, L. Herrick, C. A. Christophi, S. A. Snyder and R. Hauser, "Association between Water Consumption from Polycarbonate Containers and Bisphenol A Intake during Harsh Environmental Conditions in Summer," Environmental Science and Technology, Vol. 47, No. 7, 2013, pp. 3333-3343. doi:1 0.1021/es304038k
[46] M. K. Skinner, M. Manikkam and C. Guerrero-Bosagna, "Epigenetic Transgenerational Actions of Environmental Factors in Disease Etiology," Trends in Endocrinology and Metabolism, Vol. 21, No. 4, 2010, pp. 214-222. doi:10.1016/j.tem.2009.12.007

[47] R. M. Whyatt, X. Liu, V. A. Rauh, A. M. Calafat, A. C. Just, L. Hoepner, et al., "Maternal Prenatal Urinary Phthalate Metabolite Concentrations and Child Mental, Psychomotor, and Behavioral Development at 3 Years of Age," Environmental Health Perspectives, Vol. 120, No. 2, 2012, pp. 290-295. doi:10.1289/ehp.1103705

[48] J. Chevrier, R. B. Gunier, A. Bradman, N. T. Holland, A. M. Calafat, B. Eskenazi, et al., "Maternal Urinary Bisphenol A during Pregnancy and Maternal and Neonatal Thyroid Function in the CHAMACOS Study," Environmental Health Perspectives, Vol. 121, No. 1, 2013, pp. $138-144$

[49] B. Eskenazi, J. Chevrier, S. A. Rauch, K. Kogut, K. G. Harley, C. Johnson, et al., "In Utero and Childhood Polybrominated Diphenyl Ether (PBDE) Exposures and Neurodevelopment in the CHAMACOS Study," Environmental Health Perspectives, Vol. 121, No. 1, 2013, pp. 257-262.

[50] S. S. Andra, K. C. Makris, J. P. Shine and C. Lu, "CoLeaching of Brominated Compounds and Antimony from Bottled Water," Environment International, Vol. 38, No.1, 2012, pp. 45-53. doi:10.1016/j.envint.2011.08.007 